MATHEMATICS OF COMPUTATION

Volume 67, Number 224, October 1998, Pages 1727-1733

S $0025-5718(98) 01002-3$

\title{
INFINITE FAMILIES OF SOLUTIONS OF THE EQUATION $\left(\begin{array}{l}n \\ k\end{array}\right)=2\left(\begin{array}{l}a \\ b\end{array}\right)$
}

\author{
P. GOETGHELUCK
}

\begin{abstract}
We give explicit formulas providing two new infinite families of
\end{abstract} couples of binomial coefficients whose ratio is 2 .

\section{INTRODUCTION}

For every positive integer $r$ we have $\left(\begin{array}{c}2 r \\ r\end{array}\right)=2\left(\begin{array}{c}2 r-1 \\ r-1\end{array}\right)$, so $(n, k, a, b)=$ $(2 r, r, 2 r-1, r-1)(r=1,2,3, \ldots)$ is a family of solutions of the equation

$$
\left(\begin{array}{l}
n \\
k
\end{array}\right)=2\left(\begin{array}{l}
a \\
b
\end{array}\right)
$$

where $n, k, a, b$ are unknown integers (we can assume, without loss of generality, that $k \leq n / 2$ and $b \leq a / 2)$.

Are there any other solutions or family of solutions of equation (1)? Partial answers to this question can be found in [1], [4], [9] and [11].

It should be noted that a simple application of Siegel's theorem [7, Th. 22, p. 278], implies that for any fixed $k \geq 2$ and $b \geq 2$ with $k+b>4$, there are only finitely many solutions $n, a$ to equation (1).

D. Singmaster [10] found an infinite family of solutions of the equation $\left(\begin{array}{l}n \\ k\end{array}\right)=\left(\begin{array}{l}a \\ b\end{array}\right)$ in terms of Fibonacci numbers, and more recently B. M. M. de Weger [12] gave some complementary results, in particular on rational solutions of $\left(\begin{array}{l}n \\ 3\end{array}\right)=\left(\begin{array}{l}a \\ 4\end{array}\right)$.

In the present note the search for solutions of (1) is made using the same method as Singmaster in [10]:

1) A computer search is made, producing a list of solutions.

2) Computer solutions are examined. Some of them are proved to belong to infinite families by solving Pell's equations.

Singmaster performed his computer search for solutions of $\left(\begin{array}{l}n \\ k\end{array}\right)=\left(\begin{array}{l}a \\ b\end{array}\right)$ with the exact value of binomial coefficients. So he was limited by the size $\left(2^{48}\right)$ of integers implemented on the computer. In the present work the computations involving binomial coefficients use only the factorization of the number instead of its numerical representation:

$$
\begin{gathered}
\text { if }\left(\begin{array}{l}
n \\
k
\end{array}\right)=2^{\alpha_{1}} 3^{\alpha_{2}} 5^{\alpha_{3}} \ldots \text { (there are no primes } p>n \text { in the factorization), } \\
\text { then }\left(\begin{array}{l}
n \\
k
\end{array}\right) \text { is represented by the finite sequence }\left(\alpha_{1}, \alpha_{2}, \alpha_{3}, \ldots\right) .
\end{gathered}
$$

Received by the editor May 29, 1996 and, in revised form, October 7, 1996 .

1991 Mathematics Subject Classification. Primary 11B65, 11Y50, $11 B 37$.

(C)1998 American Mathematical Society 
Using this process, computer searches were made in the following domains, where binomial coefficients can be very big:

- $n \leq 100, a \leq 10000$,

- $n \leq 1000, a<n+1000$,

- for particular values of $k$ and $b$.

In the next section we give the main results: examining first a list of computer solutions of (1), we find three obvious infinite families of solutions, and we are led to solve Pell's equations providing two nonobvious infinite families of solutions. In Section 3, we exhibit another infinite family of solutions, which is easily deduced from the results of Section 2. Section 4 is devoted to explaining the process of factorization of binomial coefficients. In Section 5 we present and discuss some finite families of solutions.

Let us note that for any given integer $\lambda$ the equation $\left(\begin{array}{l}n \\ k\end{array}\right)=\lambda\left(\begin{array}{l}a \\ b\end{array}\right)$ can be investigated by the same method.

\section{INFINITE FAMILIES OF SOLUTIONS}

In this section a list of computer solutions of equation (1) is examined. Some of them clearly belong to obvious infinite families of solutions. Others are solutions of $\left(\begin{array}{l}n \\ 2\end{array}\right)=2\left(\begin{array}{l}a \\ 2\end{array}\right)$ and $\left(\begin{array}{l}n-1 \\ k+1\end{array}\right)=2\left(\begin{array}{l}n \\ k\end{array}\right)$. Solving completely these two equations produces two new nontrivial infinite families of solutions.

2.1. Obvious solutions. The computer search gave many numerical solutions, such as

$$
\begin{aligned}
& \left(\begin{array}{l}
2 \\
1
\end{array}\right)=2\left(\begin{array}{l}
1 \\
0
\end{array}\right), \quad\left(\begin{array}{l}
4 \\
2
\end{array}\right)=2\left(\begin{array}{l}
3 \\
1
\end{array}\right), \quad\left(\begin{array}{l}
6 \\
3
\end{array}\right)=2\left(\begin{array}{l}
5 \\
2
\end{array}\right), \quad\left(\begin{array}{l}
8 \\
4
\end{array}\right)=2\left(\begin{array}{l}
7 \\
3
\end{array}\right), \ldots \\
& \left(\begin{array}{l}
4 \\
1
\end{array}\right)=2\left(\begin{array}{l}
2 \\
1
\end{array}\right), \quad\left(\begin{array}{l}
6 \\
3
\end{array}\right)=2\left(\begin{array}{c}
10 \\
1
\end{array}\right), \quad\left(\begin{array}{l}
8 \\
2
\end{array}\right)=2\left(\begin{array}{c}
14 \\
1
\end{array}\right), \quad\left(\begin{array}{l}
8 \\
3
\end{array}\right)=2\left(\begin{array}{c}
28 \\
1
\end{array}\right), \ldots \\
& \left(\begin{array}{l}
2 \\
1
\end{array}\right)=2\left(\begin{array}{l}
2 \\
0
\end{array}\right), \quad\left(\begin{array}{l}
5 \\
2
\end{array}\right)=2\left(\begin{array}{l}
5 \\
1
\end{array}\right), \quad\left(\begin{array}{l}
8 \\
3
\end{array}\right)=2\left(\begin{array}{l}
8 \\
2
\end{array}\right), \quad\left(\begin{array}{c}
11 \\
4
\end{array}\right)=2\left(\begin{array}{c}
11 \\
3
\end{array}\right), \ldots
\end{aligned}
$$

belonging to the three obvious infinite families of solutions described by the following formulas:

$$
\begin{gathered}
\left(\begin{array}{c}
2 r \\
r
\end{array}\right)=2\left(\begin{array}{c}
2 r-1 \\
r-1
\end{array}\right) \quad(r=1,2, \ldots), \\
\left(\begin{array}{c}
n \\
k
\end{array}\right)=2 r=2\left(\begin{array}{c}
r \\
1
\end{array}\right) \quad(r=1,2, \ldots), \\
\left(\begin{array}{c}
3 r-1 \\
r
\end{array}\right)=2\left(\begin{array}{c}
3 r-1 \\
r-1
\end{array}\right) \quad(r=1,2, \ldots) .
\end{gathered}
$$

2.2. Infinite family of solutions of $\left(\begin{array}{l}n \\ 2\end{array}\right)=2\left(\begin{array}{l}a \\ 2\end{array}\right)$. By computer search we have found the following three numerical solutions:

$$
\left(\begin{array}{c}
21 \\
2
\end{array}\right)=2\left(\begin{array}{c}
15 \\
2
\end{array}\right), \quad\left(\begin{array}{c}
120 \\
2
\end{array}\right)=2\left(\begin{array}{c}
85 \\
2
\end{array}\right), \quad\left(\begin{array}{c}
697 \\
2
\end{array}\right)=2\left(\begin{array}{c}
493 \\
2
\end{array}\right) .
$$

Thus, we are led to wonder if the equation $\left(\begin{array}{l}n \\ 2\end{array}\right)=2\left(\begin{array}{l}a \\ 2\end{array}\right)$ has any other solution.

We put $a=n-c(0<c<n)$. The equation $\left(\begin{array}{c}n \\ 2\end{array}\right)=2\left(\begin{array}{c}n-c \\ 2\end{array}\right)$ is equivalent to $n^{2}-n(4 c+1)+2\left(c^{2}+c\right)=0$, giving $n=\left(4 c+1+\sqrt{8 c^{2}+1}\right) / 2($ since $n>c)$. 
Thus $n$ is an integer if and only if $8 c^{2}+1$ is a square, and we must solve the Pell's equation

$$
x^{2}-8 c^{2}=1 .
$$

The least positive integers satisfying $(2)$ are $(x, c)=(3,1)$, and then (see [8, Th. 4.4, p. 118]) all positive solutions $\left(x_{i}, c_{i}\right)$ of $(2)$ are given by

$$
x_{i}+c_{i} \sqrt{8}=(3+\sqrt{8})^{i} \quad(i=1,2,3, \ldots)
$$

or equivalently

$$
x_{1}=3, \quad c_{1}=1, \quad x_{i+1}=3 x_{i}+8 c_{i}, \quad c_{i+1}=x_{i}+3 c_{i} \quad(i=1,2,3 \ldots) .
$$

By a straightforward calculation, the following induction formulas provide all solutions $\left(n_{i}, a_{i}\right)$ of the equation $\left(\begin{array}{c}n \\ 2\end{array}\right)=2\left(\begin{array}{c}a \\ 2\end{array}\right)$ :

$$
\begin{gathered}
\left(n_{1}, a_{1}\right)=(4,3), \quad\left(n_{2}, a_{2}\right)=(21,15), \\
\left(n_{i+2}, a_{i+2}\right)=6\left(n_{i+1}, a_{i+1}\right)-\left(n_{i}, a_{i}\right)-(2,2) \quad(i=1,2,3, \ldots) .
\end{gathered}
$$

The computer search found the first four solutions $(4,3),(21,15),(120,85)$, $(697,493)$; the next are $(4060,2871),(23661,16731), \ldots$.

2.3. Infinite family of solutions of $\left(\begin{array}{l}n-1 \\ k+1\end{array}\right)=2\left(\begin{array}{l}n \\ k\end{array}\right)$. The computer search also gave the following two numerical solutions:

$$
\left(\begin{array}{l}
43 \\
12
\end{array}\right)=2\left(\begin{array}{l}
44 \\
11
\end{array}\right), \quad\left(\begin{array}{l}
614 \\
165
\end{array}\right)=2\left(\begin{array}{l}
615 \\
164
\end{array}\right)
$$

leading to the study of the equation $\left(\begin{array}{c}n-1 \\ k+1\end{array}\right)=2\left(\begin{array}{l}n \\ k\end{array}\right)$.

This equation is equivalent to $n^{2}-n(4 k+3)+\left(k^{2}+k\right)=0$, whose solution satisfying $n>k$ is $n=\left(4 k+3+\sqrt{12 k^{2}+20 k+9}\right) / 2$.

Thus $n$ is an integer if and only if $12 k^{2}+20 k+9$ is a square, and we are led to solve

$$
12 k^{2}+20 k+9=x^{2} .
$$

The positive solution of the last equation is $k=\left(-5+\sqrt{3 x^{2}-2}\right) / 6$, and $k$ is an integer if and only if

$$
3 x^{2}-2=y^{2}, \quad y \equiv 5(\bmod 6) .
$$

Since $(2,1)$ and $(1,1)$ are the least positive solutions of the equations $z^{2}-3 t^{2}=1$ and $z^{2}-3 t^{2}=-2$, respectively, an infinite family of solutions $\left(z_{i}, t_{i}\right)$ of $z^{2}-3 t^{2}=-2$ (see $[6$, Th. 8.8, p. 148]) is given by

$$
z_{i}+t_{i} \sqrt{3}=(1+\sqrt{3})(2+\sqrt{3})^{i} \quad(i=0,1,2, \ldots)
$$

or equivalently

$$
\begin{gathered}
\left(z_{0}, t_{0}\right)=(1,1), \quad\left(z_{1}, t_{1}\right)=(5,3), \\
\left(z_{i+2}, t_{i+2}\right)=4\left(z_{i+1}, t_{i+1}\right)-\left(z_{i}, t_{i}\right) \quad(i=0,1,2, \ldots) .
\end{gathered}
$$

These induction formulas show that $z_{i} \equiv(-1)^{i}(\bmod 6)$. Then an infinite family of solutions of $(3)$ is $\left(y_{i}, x_{i}\right)=\left(z_{2 i-1}, t_{2 i-1}\right)(i=1,2,3, \ldots)$, which can be described by

$$
\begin{gathered}
\left(y_{1}, x_{1}\right)=(5,3), \quad\left(y_{2}, x_{2}\right)=(71,41), \\
\left(y_{i+2}, x_{i+2}\right)=14\left(y_{i+1}, x_{i+1}\right)-\left(y_{i}, x_{i}\right) \quad(i=1,2,3, \ldots) .
\end{gathered}
$$


The corresponding solutions $\left(n_{i}, k_{i}\right)$ of $\left(\begin{array}{l}n-1 \\ k+1\end{array}\right)=2\left(\begin{array}{l}n \\ k\end{array}\right)$ satisfy $k_{i}=\left(y_{i}-5\right) / 6$ and $n_{i}=\left(4 k_{i}+3+x_{i}\right) / 2$, and then are given by

$$
\begin{gathered}
\left(n_{1}, k_{1}\right)=(3,0), \quad\left(n_{2}, k_{2}\right)=(44,11), \\
\left(n_{i+2}, k_{i+2}\right)=14\left(n_{i+1}, k_{i+1}\right)-\left(n_{i}, k_{i}\right)+(2,10) \quad(i=1,2,3, \ldots) .
\end{gathered}
$$

The computer search found $\left(n_{1}, k_{1}\right)=(3,0),\left(n_{2}, k_{2}\right)=(44,11)$, and $\left(n_{3}, k_{3}\right)=$ $(615,164)$. The next two solutions are $(8568,2295)$ and $(119339,31976)$.

\section{ANOTHER INFINITE FAMILY OF SOLUtions}

3.1. Equivalent solutions. If for some $n, a$ and $k$ we have $\left(\begin{array}{l}n \\ k\end{array}\right)=2\left(\begin{array}{l}a \\ k\end{array}\right)$, then multiplying both sides by $\frac{k !(n-k) !}{a !(n-a) !}$ yields

$$
\left(\begin{array}{c}
n \\
n-a
\end{array}\right)=2\left(\begin{array}{l}
n-k \\
n-a
\end{array}\right) .
$$

We say that corresponding solutions $(n, k, a, k)$ and $(n, n-a, n-k, n-a)$ are equivalent.

3.2. Application. We have found the following three numerical solutions

$$
\left(\begin{array}{c}
21 \\
6
\end{array}\right)=2\left(\begin{array}{c}
19 \\
6
\end{array}\right), \quad\left(\begin{array}{c}
120 \\
35
\end{array}\right)=2\left(\begin{array}{c}
118 \\
35
\end{array}\right), \quad\left(\begin{array}{l}
697 \\
204
\end{array}\right)=2\left(\begin{array}{l}
695 \\
204
\end{array}\right)
$$

which are clearly equivalent to the solutions

$$
\left(\begin{array}{c}
21 \\
2
\end{array}\right)=2\left(\begin{array}{c}
15 \\
2
\end{array}\right), \quad\left(\begin{array}{c}
120 \\
2
\end{array}\right)=2\left(\begin{array}{c}
85 \\
2
\end{array}\right), \quad\left(\begin{array}{c}
697 \\
2
\end{array}\right)=2\left(\begin{array}{c}
493 \\
2
\end{array}\right)
$$

of subsection 2.2 .

More generally, if $(n, k, a, b)=(n, 2, a, 2)$ is a solution of $\left(\begin{array}{l}n \\ k\end{array}\right)=2\left(\begin{array}{l}a \\ b\end{array}\right)$, then $(n, n-a, n-2, n-a)$ is another solution of this equation. So the infinite family of solutions found in subsection 2.2 provides a new infinite family $\left(n_{i}, u_{i}, n_{i}-2, u_{i}\right)$ of equivalent solutions given by the following induction formulas:

$$
\begin{gathered}
\left(n_{1}, u_{1}\right)=(4,1), \quad\left(n_{2}, u_{2}\right)=(21,6), \\
\left(n_{i+2}, u_{i+2}\right)=6\left(n_{i+1}, u_{i+1}\right)-\left(n_{i}, u_{i}\right)-(2,0) \quad(i=1,2,3, \ldots) .
\end{gathered}
$$

The computer search found the first four solutions $(4,1),(21,6),(120,35),(697,204)$; the next are $(4060,1189),(23661,6930), \ldots$.

\section{EXPANSION OF A BINOMIAL COEFFICIENT INTO PRIMES}

We denote by $E_{p}(n, k)$ the power of the prime $p$ in the expansion of $\left(\begin{array}{l}n \\ k\end{array}\right)$ into primes. The basic result is the following:

Theorem (Kummer, 1852 [5, p. 115]). For any prime $p$ and any integers $n$ and $k$ $(0 \leq k \leq n), E_{p}(n, k)$ is equal to the number of borrow $(s)$ in the subtraction $n-k$ in base $p$.

Some immediate consequences make Kummer's theorem easy to apply:

- If $p>n$, then $E_{p}(n, k)=0$.

- If $2 k \leq n$ and $n-k<p \leq n$, then $E_{p}(n, k)=1$.

- If $2 k \leq n$ and $\frac{n}{2}<p \leq n-k$, then $E_{p}(n, k)=0$.

- If $2 k \leq n$ and $\sqrt{n}<p \leq \frac{n}{2}$, then $E_{p}(n, k)=0$ or 1 , and $E_{p}(n, k)=1$ if and only if $n \bmod p<k \bmod p$. 


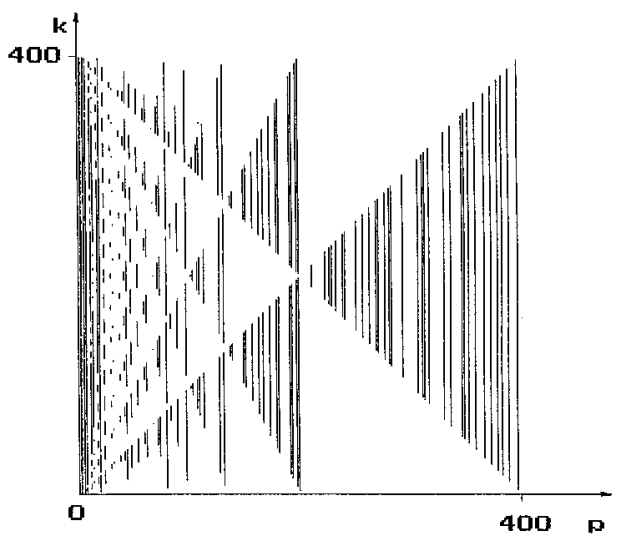

Figure 1. The set of all $(p, k)$ such that $p$ is prime and divides $\left(\begin{array}{c}400 \\ k\end{array}\right)$

To get the expansion of $\left(\begin{array}{l}n \\ k\end{array}\right)$ into primes we need a table of primes up to $n$. Then, under the assumption $n \geq 4$ and $2 k \leq n$, the computation is made according to the following scheme:

$$
\begin{array}{rlrl}
\text { if } 2<p \leq \sqrt{n}, & E_{p}(n, k) & =\text { number of borrow }(\mathrm{s}) \\
& & & \text { in the subtraction } n-k \text { in base } p ; \\
\text { if } \sqrt{n}<p \leq n / 2, & E_{p}(n, k) & =0 \text { if } k \bmod p \leq n \bmod p \text { and } \\
& E_{p}(n, k)=1 \text { if } k \bmod p>n \bmod p ; \\
\text { if } n / 2<p \leq n-k, & E_{p}(n, k)=0 ; \\
\text { if } n-k<p \leq n, & E_{p}(n, k)=1 ; \\
\text { if } n<p, & E_{p}(n, k)=0 .
\end{array}
$$

A detailed algorithm for computing the factorization of $\left(\begin{array}{l}n \\ k\end{array}\right)$ into primes can be found in [2].

Using a PC with a $66 \mathrm{mhz} 486 \mathrm{CPU}$, the computation of the factorization of $\left(\begin{array}{l}n \\ k\end{array}\right)$ spends less than $3 \cdot 10^{-4}$ second if $n \leq 1000$ and less than $2 \cdot 10^{-3}$ second if $n \leq 10000$.

Application. 1) Obviously, in the computer search, instead of comparing values, we compare the factorizations of $\left(\begin{array}{l}n \\ k\end{array}\right)$ and $2\left(\begin{array}{l}a \\ b\end{array}\right)$.

2) Suppose that for given $n$ and $k$, we search for a solution of (1) with $a \leq n$. If there are primes in the interval $(n-k, n]$, let $p$ be the greatest of them. By the second consequence of Kummer's theorem, $p$ divides $\left(\begin{array}{l}n \\ k\end{array}\right)$, and therefore, $p$ divides $\left(\begin{array}{l}a \\ b\end{array}\right)$. Then $p \leq a \leq n$. If we now search for a solution of (1) with $a>n$, an analogous proof shows that for any prime $q$ satisfying $n<q \leq a$ we must have $b \leq a-q$.

Then the computer search is restricted to very few binomial coefficients.

Figure 1 gives a geometric illustration of these results. For a given $n$ (here $n=400)$, the figure is drawn by plotting every $(p, k)$ such that $p$ is prime and divides $\left(\begin{array}{l}n \\ k\end{array}\right)$. (The structure of the pattern, which is the same for any $n$, is explained in [3]). So, to get the list of primes $p$ dividing $\left(\begin{array}{l}n \\ k\end{array}\right)$ we need only to draw a horizontal line at ordinate $k$ and collect abscissas where it meets vertical segments.

These remarks explain why we find only very few solutions to equation (1). 


\section{Finite families of SOlutions}

5.1. A list of solutions. Besides the members of infinite families of solutions of Sections 2 and 3, the numerical computation also found that:

$$
\begin{aligned}
\text { a) }\left(\begin{array}{c}
36 \\
3
\end{array}\right) & =2\left(\begin{array}{c}
85 \\
2
\end{array}\right),\left(\begin{array}{c}
10 \\
4
\end{array}\right)=2\left(\begin{array}{c}
15 \\
2
\end{array}\right),\left(\begin{array}{c}
11 \\
5
\end{array}\right)=2\left(\begin{array}{c}
22 \\
2
\end{array}\right) \\
\left(\begin{array}{c}
25 \\
5
\end{array}\right) & =2\left(\begin{array}{c}
231 \\
2
\end{array}\right),\left(\begin{array}{c}
30 \\
5
\end{array}\right)=2\left(\begin{array}{c}
378 \\
2
\end{array}\right),\left(\begin{array}{c}
34 \\
5
\end{array}\right)=2\left(\begin{array}{c}
528 \\
2
\end{array}\right) \\
\left(\begin{array}{c}
38 \\
7
\end{array}\right) & =2\left(\begin{array}{c}
3553 \\
2
\end{array}\right),\left(\begin{array}{c}
18 \\
9
\end{array}\right)=2\left(\begin{array}{c}
221 \\
2
\end{array}\right) \\
\text { b) }\left(\begin{array}{c}
85 \\
34
\end{array}\right) & =2\left(\begin{array}{c}
83 \\
35
\end{array}\right) ; \\
\text { c) }\left(\begin{array}{c}
45 \\
2
\end{array}\right) & =2\left(\begin{array}{c}
12 \\
4
\end{array}\right),\left(\begin{array}{c}
273 \\
2
\end{array}\right)=2\left(\begin{array}{c}
18 \\
6
\end{array}\right) .
\end{aligned}
$$

These solutions are listed here according to the following classification:

Type a. Solutions $\left(\begin{array}{l}n \\ k\end{array}\right)=2\left(\begin{array}{l}a \\ 2\end{array}\right)$.

Type b. Solutions $\left(\begin{array}{l}n \\ k\end{array}\right)=2\left(\begin{array}{c}n-2 \\ k+1\end{array}\right)$.

Type c. Solutions $\left(\begin{array}{c}n \\ 2\end{array}\right)=2\left(\begin{array}{c}6 b \\ 2 b\end{array}\right)$.

Special programs have been written to exhibit other results of these three types. None of them gave any new solution.

\subsection{Remarks.}

5.2.1. We have no explanation of the fact that there are so many solutions of type $a$. However we remark that for these solutions $\left(\begin{array}{l}a \\ 2\end{array}\right)$ and $n$ have "many" prime factors.

5.2.2. If we take $u=n-k-1$ and $v=k+1$, then $\left(\begin{array}{l}n \\ k\end{array}\right)=2\left(\begin{array}{l}n-2 \\ k+1\end{array}\right)$ is equivalent to the equation $2 u^{3}-v u^{2}-\left(2 v^{2}-v+2\right) u-\left(v^{3}-v^{2}\right)=0$ or $2 u^{3}-v u^{2}-2 v^{2} u-v^{3}=$ $2 u-u v-v^{2}$. The fact that the polynomial $2 x^{3}-x^{2}-2 x-1$ has no rational roots implies that there are only finitely many solutions of type $b$ (this is an application of the following result [7, p. 278] established by Schinzel: let $f$ and $g$ be two polynomials with integer coefficients satisfying $\operatorname{deg}(f)>2, \operatorname{deg}(g)<\operatorname{deg}(f)$ and $f$ irreducible in the rational field; then the equation $f(u, v)=g(u, v)$ has only a finite number of integer solutions).

The computation shows that $\left(\begin{array}{l}85 \\ 34\end{array}\right)=2\left(\begin{array}{l}83 \\ 35\end{array}\right)$ is the only solution of type $b$ for $n \leq 10^{6}$.

5.2.3. Since $\left(\begin{array}{l}n \\ 2\end{array}\right)=2\left(\begin{array}{l}a \\ 2\end{array}\right)$ gave many solutions, we have investigated the equation $\left(\begin{array}{l}n \\ k\end{array}\right)=2\left(\begin{array}{l}a \\ k\end{array}\right)(k>2)$. This equation has only a finite number (possibly zero) of solutions, and, as seen in subsection 3.1 , is equivalent to the equation

$$
\left(\begin{array}{c}
n \\
n-a
\end{array}\right)=2\left(\begin{array}{l}
n-k \\
n-a
\end{array}\right)
$$

Then, when seeking solutions, we can assume that $n-a<k$.

A computer search shows that there are no solutions in the domains

- $3 \leq k \leq 40, n \leq 10^{6}, n-a<k$;

- $n \leq 30000, n-a \leq k$. 


\section{REFERENCES}

1. A. D. Barry, L'équation diophantienne $x(x+1)=k y(y+1)$, Enseign. Math. (2) 25 (1979), 23-31. MR 81b:10006

2. P. Goetgheluck, Computing binomial coefficients, Amer. Math. Monthly 94 (1987), 360-365.

3. _ On prime divisors of binomial coefficients, Math. Comp., 51 (1988), 325-329. MR 89f: 11033

4. C. G. Khatri and A. M. Vaidya, On integers which are ratios of two triangular numbers, J. Indian Math. Soc. (N.S.) 35 (1971), 205-215. MR 46:7157

5. E. E. Kummer, Uber die Erganzumgssatze zu den allgemeinen Reciprocität gesetzen, J. Reine Angew. Math. 44 (1852), 93-164.

6. W. J. Le Veque, Topics in Number Theory, Vol. 1, Addison-Wesley, Reading, MA, 1956. MR 18:283d

7. L. J. Mordell, Diophantine Equations, Academic Press, London, 1969. MR 40:2600

8. C. D. Olds, Continued Fractions, Random House, New York, 1963. MR 26:3672

9. T. N. Shorey, On the ratio of values of a polynomial, Proc. Indian Acad. Sci. (Math. Sci.) 93 (1984), 109-116. MR 87c:11037

10. D. Singmaster, Repeated binomial coefficients and Fibonacci numbers, Fibonacci Quarterly 13 (1975), 295-298. MR 54:224

11. S. Thouvenot, Résolution en nombres entiers de l'équation diophantienne $n(n+1)=$ $2 n^{\prime}\left(n^{\prime}+1\right)$, Enseign. Math. (2) 16 (1970), 203-217. MR 45:3319

12. B. M. M. de Weger, Equal binomial coefficients: Some elementary considerations, J. Number Theory, 63 (1997), 373-386. MR 98b:11027

Université de Paris-Sud, Mathématiques, Bat 425, 91405 Orsay Cedex, France

E-mail address: goetghe@iut-orsay.fr 